\title{
Deep CCD Surface Photometry of the Edge-On Spiral NGC 4244
}

\author{
Anne M. Fry \\ Department of Astronomy, Case Western Reserve University, Cleveland OH 44106-7215 \\ electronic mail: anne@smaug.astr.cwru.edu \\ Heather L. Morrison円 \\ Department of Astronomy and Department of Physics, Case Western Reserve University, \\ Cleveland $\mathrm{OH}$ 44106-7215 \\ electronic mail: heather@vegemite.astr.cwru.edu \\ Paul Harding \\ Steward Observatory, University of Arizona, Tucson, Arizona 85726 \\ electronic mail: harding@as.arizona.edu \\ and \\ Todd A. Boroson \\ US Gemini Program, National Optical Astronomy Observatories[t, Tucson, Arizona 85726 \\ electronic mail: tyb@noao.edu
}

\begin{abstract}
We have obtained deep surface photometry of the edge-on spiral galaxy NGC 4244. Our data reliably reach $27.5 R$ magnitude $\operatorname{arcsec}^{-2}$, a significant improvement on our earlier deep CCD surface photometry of other galaxies. NGC 4244 is a nearby Scd galaxy whose total luminosity is approximately one magnitude fainter than the peak of the Sc luminosity function. We find that it has a simple structure: a single exponential disk, with a scale height $h_{Z}=246$ $\pm 2 \mathrm{pc}$, a scale length $h_{R}=1.84 \pm 0.02 \mathrm{kpc}$ and a disk cutoff at a radius $R_{\max }$ $=10.0 \mathrm{kpc}$ (5.4 scale lengths). We confirm a strong cutoff in the stellar disk at $R_{\text {max }}$, which happens over only $1 \mathrm{kpc}$. We do not see any statistically significant evidence for disk flaring with radius. Unlike the more luminous Sc galaxies NGC 5907 and M 33, NGC 4244 does not show any evidence for a second component, such as a thick disk or halo, at $\mu_{R}<27.5$ magnitude $\operatorname{arcsec}^{-2}$.

Keywords: galaxies: individual (NGC 4244) — galaxies: photometry — galaxies: spiral
\end{abstract}

\footnotetext{
${ }^{1}$ Cottrell Scholar of Research Corporation and NSF CAREER fellow

${ }^{2}$ The National Optical Astronomy Observatories are operated by the Association of Universities for Research in Astronomy, Inc (AURA) under cooperative agreement with the National Science Foundation
} 


\section{INTRODUCTION}

In order to better characterize the distribution of luminosity in the stellar components of disk galaxies, we are conducting a detailed study of edge-on disk galaxies with a range of bulge-to-disk ratio using deep CCD surface photometry. Our galaxies range in Hubble type from S0 to Scd, and in bulge-to-disk-ratio from 20 (NGC 3115) to 0.03 (NGC 4244, this work \& Baggett et al. 1998). We will re-examine the structure of their bright disks and bulges, and also study, for the first time, the structure of faint outer components such as thick disks and stellar halos.

These faint outer components are important because they may trace the history of the galaxy, either because they contain the oldest stars (Eggen et al. 1962, Edvardsson et al. 1993 ) or because they were formed via accretions of smaller galaxies (Searle \& Zinn 1978, Davis et al. 1985, Quinn \& Goodman 1986). In addition, if they are made of old stars, they should provide connections to high-z galaxy observations, both direct (Steidel et al. 1996) and indirect via absorption line studies (Wolfe 1990).

The simplest galaxies structurally are those of late type - Sc and later. To first order, they comprise simply a disk with exponential surface brightness falloff (Freeman 1970, van der Kruit \& Searle 1981). We have already studied a large Sc galaxy, NGC 5907 (Morrison et al. 1994). Our analysis confirmed previous findings on its thin disk (van der Kruit \& Searle 1981), but we found an unusual second component, whose luminosity falls off very slowly with radius (see Sackett et al. 1994, Monkiewicz et al. 1999). Since the galaxy luminosity function (LF) for Sc galaxies is very broad, and peaked at total blue luminosity $\left(M_{B, t o t}\right)$ of -19.5 (Binggeli et al. 1988), making NGC $5907\left(M_{B, t o t}=-20.55\right.$, Tully 1988) an unusually luminous Sc galaxy, we have also studied NGC 4244, a smaller late-type spiral whose total magnitude of $-18.4 B$ mag (Olling 1996a) is almost a magnitude below the broad peak of the field Sc LF. NGC 4244 has Hubble type Scd, is located 3.6 Mpc away (Tully-Fisher, Aaronson et al. 1986), and has a flat rotation curve with $V_{\text {rot }}=100 \mathrm{~km} / \mathrm{s}$ (Olling 1996a). Beyond the optical disk, NGC 4244 has a declining rotation curve (Olling 1996a).

In this paper we present deep surface photometry of NGC 4244. Our data reach

reliably to $\mu_{R}=27.5$ magnitude $\operatorname{arcsec}^{-2}$, significantly fainter than our previous published data on NGC 5907. Questions that we will address using this extremely deep data are:

- do small late-type disk galaxies such as NGC 4244 have similar properties to large ones such as NGC 5907?

- what are the properties of NGC 4244's luminous thin disk? Does the stellar disk 
have a distinct edge? What is its vertical scale height? Does this scale height remain constant with radius?

- does NGC 4244 show fainter components like a thick disk or halo, or like NGC 5907's unusual second component?

NGC 4244 is less massive than a typical Sc: the total mass of NGC 4244 is $9.77 \mathrm{x}$ $10^{9} M_{\odot}$ (Tully 1988), whereas the average total mass of an Sc is $\approx 10^{11} M_{\odot}$ (Roberts \& Haynes 1994). However the H I mass fraction $M_{H I} / M_{t o t}$ (Olling 1996a) is fairly typical for an Sc galaxy: 0.14 . The mean $M_{H I} / M_{t o t}$ for a typical Sc (from Roberts \& Haynes 1994) is 0.1 . The mass fraction of $\mathrm{H}_{2}\left(M_{H_{2}} / M_{\text {tot }}\right)$ is low in NGC 4244, relative to other galaxies of the same mass. In his CO study, Sage (1993) found $\left\langle M_{H 2} / M_{T}\right\rangle=0.0013$, which placed NGC 4244 in his mass class III, for which $\left\langle M_{H 2}\right\rangle=8.4 \times 10^{7} M_{\odot}$ or $<M_{H 2} / M_{T}>\approx 0.008$. The current star formation rate in NGC 4244 is also low, as can be seen from its low X-ray, radio continuum, and IRAS emission (cf. Olling 1996a).

The Hubble type of a galaxy is known to correlate roughly with its environment in the sense that late-type galaxies are found in more isolated areas (Dressler 1980). In addition, we might expect to see a correlation between the existence of thick disks and galaxy environment if the accretion theory of thick disk formation is correct. NGC 4244 lies in the Coma-Centaurus spur, and is thought to be a member of the CVn I de Vaucouleurs 1975) or B4 (Kraan-Kortweg \& Tammann 1979) group. Its small distance makes group membership very difficult to decide, since group peculiar velocities are of the same size as the Hubble flow. We have plotted in Figure 1 supergalactic coordinates of galaxies in the B4 and nearby B5 groups of Kraan-Kortweg \& Tammann - it is not clear whether these two groups are separate entities. We can only conclude from these data that NGC 4244 lives in a relatively isolated environment, with only three galaxies of similar or greater brightness within $\sim 2 \mathrm{Mpc}(\mathrm{NGC} 4449, \mathrm{NGC} 4736$, NGC 4826), the brightest of which (NGC 4736) has $\mathrm{M}_{B, t o t}=-19.37 \mathrm{mag}, 1.7 \mathrm{mag}$ brighter than NGC 4244 .

van der Kruit \& Searle (1981a) used photographic surface photometry of NGC 4244 to study its structure, finding the disk well modeled by a radial exponential function and a vertical sech $^{2}$ function, with a sharp cutoff at 5.3 radial scale lengths. They found no evidence for a thick disk, in contrast to their studies of disk galaxies with larger B/D ratios where thick disks were seen. However, thick disks are very faint, compared to both the night sky and the light of the younger, thin disks, and, thus, detection and accurate determinations of the properties of thick disks from photographic work are difficult. The improved precision of CCDs is needed, and our faint CCD photometry of NGC 4244 will put much stronger limits on the existence of a thick disk in this galaxy. 
The flaring of a galaxy's stellar disk gives constraints on the balance between the gravitational potential the disk experiences and heating mechanisms the stars experience. van der Kruit \& Searle (1981a,b,1982a,b) found that stellar disks in the galaxies they studied had constant scale heights with radius, although this has recently been challenged (de Grijs \& Peltier 1997). NGC 4244 is a good galaxy in which to study disk flaring, as it has no large companions and no apparent thick disk (cf. van der Kruit \& Searle 1981a). We will discuss the strong limits our data place on any variation of disk scale height with radius.

\section{OBSERVATIONS \& REDUCTIONS}

The NGC 4244 data were obtained over a five night run in March 1997 and a six night run in April 1997 using the 24" $/ 36^{\prime \prime}$ Burrell Schmidt telescope [5 $^{3}$ at Kitt Peak National Observatoryf. The images were taken using a 2048x2048 frontside-illuminated Tektronix CCD (S2KA) mounted at Newtonian focus. With this setup, the field of view is 69 square arcmin, with each $21 \mu \mathrm{m}$ pixel imaging 2."03 of sky. The gain was set to $2.5 \mathrm{e}^{-} / \mathrm{ADU}$ and the readout noise was $3 \mathrm{e}^{-}(1.2 \mathrm{ADU})$. All exposures were made through a Harris $R$ filter.

Observations were performed in the manner described by Morrison et al. (1997). Half of the time was used observing the galaxy and the other half was used to obtain dark sky flats. The dark sky flat images were taken at approximately the same hour angle and declination as the galaxy images. Over the course of the two observing runs, 66 galaxy images and 70 dark sky flats were obtained. For all of the galaxy and sky images, the exposure time was $600 \mathrm{~s}$. Conditions were photometric throughout the observing runs.

Overscan removal and bias subtraction were done in the standard manner. Then "master" sky flats were constructed from the dark sky images from each run. The individual sky flats were dithered by $\sim 5^{\circ}$, so that wings from bright stars in the field would not appear in the final, combined image. To construct the master sky flat, we used an iterative procedure. We scaled the individual sky images by their mode. Prescaling was important because, even during photometric conditions, the sky brightness varied by $\sim 0.3$ mag. This was likely due to the long tail to high wavelength of the Harris (Cousins) $R$ filter, which

\footnotetext{
${ }^{3}$ Observations made with the Burrell Schmidt of the Warner and Swasey Observatory, Case Western Reserve University.

${ }^{4}$ Kitt Peak National Observatory is a division of the National Optical Astronomy Observatories (NOAO), which are operated by the Association of Universities for Research in Astronomy, Inc. (AURA) under cooperative agreement with the National Science Foundation
} 
includes some of the strong airglow lines, combined with the change in sky brightness with zenith distance. Then, we combined the individual, scaled, sky images, using IRAF's ccdclip algorithm, which uses CCD properties to estimate expected variations in data values and removed pixels which differed from the median by more than $2 \sigma$. Each of the individual sky frames was divided by the flatfield frame to reduce the width of the distribution of sky values and so make rejection of outliers due to faint stars and stellar wings more accurate. A plane was fit to each of the individual flat-fielded sky frames and subtracted from the sky frame. The modes were recalculated, and the entire procedure repeated. This procedure was repeated until no improvement was seen in the master sky flat (ten times in this case).

The 66 galaxy images were dithered by $\sim 400^{\prime \prime}$, much less than for the sky flats. The individual galaxy images were flatfielded using the master sky flat and registered. We combined the individual galaxy images, using a median. In Figure 2 we show our final galaxy image, after subtracting a best fit plane sky and correcting to airmass 1.

One Landolt standard star field (Landolt 1992) was observed each night, giving us 26 well-exposed standard stars with a range in color and airmass. A photometric zero point of $R=22.23 \pm 0.03$ magnitude $\operatorname{arcsec}^{-2}$ corresponding to $1 \mathrm{ADU} / \mathrm{sec} /$ pixel was determined. For a $600 \mathrm{~s}$ exposure, this yields $R=29.18 \pm 0.03$ magnitude $\operatorname{arcsec}^{-2}$ corresponding to 1 ADU/exposure/pixel. As our exposures were only taken in one filter, we cannot add a color term to our calibration but estimate its magnitude as less than 0.10 mag over the color range of our standards (see Figure 3, $B-V=-0.06$ to $1.66 \mathrm{mag}, V-R=0.05$ to 0.8 ). In

fact, over the expected color range of the relatively old populations we will be studying, the color term is well below the uncertainty in the zero point.

The average sky brightness, 1998.0 ADU / pixel (see Section 3.2), corresponds to $R_{\text {sky }}$ $=20.9$ magnitude $\operatorname{arcsec}^{-2}$.

\section{ANALYSIS \& RESULTS}

For simplification in subsequent analyses, the galaxy has been rotated counter-clockwise by $42^{\circ}$. This agrees well with Olling's (1996a) determination of $-48^{\circ}$ for the position angle of NGC 4244. 


\section{1. $\quad$ Masking}

In order to reach the faintest possible surface brightness level in the galaxy, we masked out the foreground stars and background galaxies in our field. To characterize the stars we used DAOphot (Stetson 1987) to determine the positions and magnitudes of all of the stars within the frame. Using 48 bright, but unsaturated, stars, we determined the point spread function (PSF) for stars in the frame. We followed the method described in Stetson (1987), iterating until a clean subtraction within a 15x15 pixel box centered on the PSF star was obtained. Our aim was not to determine accurate photometry of all of the stars within the frame, but to mask the stars out to a constant level in surface brightness. This means that we paid particular attention to the outer wings of our PSF. We used this PSF to construct a radial profile and masked all pixels for which a foreground star contributed at least 1 ADU. The stellar radial profile was well determined out to the PSF radius, 15 pixels $\left(30^{\prime \prime}\right)$. The PSF is axisymmetric and varies along the chip. The variation is most significant for the central regions of the stars and does not strongly affect the outer wings of the stars, which are formed by processes such as scattering in the atmosphere and telescope. We describe the PSF beyond 15 pixels in Section 3.4.

Background galaxies and undetected stars were masked by hand using the IRAF task imedit. Special care was taken with stars near the galaxy, where the galaxy's luminosity gradient interferes with DAOphot's sky fitting algorithm.

NGC 4244 has a small dust lane and several visible H II regions. To mask these, a dust mask 20 pixels wide, running along the major axis, was created. In Figure 4 we show the galaxy with our final mask superimposed.

Using this mask, the data were binned up into regions whose size varied from a resolution element near the galaxy's center to 50 x 50 pixels in the regions far from its center. This procedure used a robust averaging scheme to compensate for any unmasked stars, galaxies, etc. and is described in detail in Morrison et al. (1994).

\subsection{Sky Subtraction}

Accurate sky subtraction is crucial for the faint isophotes. To determine the sky level, we used the masked image and made a histogram of sky values in bins well away from the galaxy, using bins with more than 100 pixels after masking. There are 310 such bins. Using a histogram of the sky values within these bins, we determined the sky value to be 1998.0 \pm 0.8 ADU. In Figure 5, we show this histogram. The width of the histogram provides a measure of the contribution of unmasked stars to the sky level. 


\subsection{Limits to Our Precision}

Our photometry reaches reliably to 27.5 magnitude $\operatorname{arcsec}^{-2}$. The error model is described in Appendix A. To illustrate, we work through the errors in a $13 \times 3$ pixel bin, centered 12 pixels $(425 \mathrm{pc})$ from the major axis and 150 pixels $(5.3 \mathrm{kpc})$ from the minor axis. In this bin, the mean number of counts is $\bar{C}=2498 \mathrm{ADU}$ per pixel, with the mean number of counts from the galaxy alone $\bar{g}=500 \mathrm{ADU}$ per pixel. The errors are summarized in Table 1. The dominant source of error in the thin disk region of the galaxy is the error arising from surface brightness fluctuations within NGC 4244, because NGC 4244 is so close to the Milky Way (its distance is $3.6 \mathrm{Mpc}$; note that it is marginally resolved even from the ground.) Surface brightness fluctuations dominate the formal errors wherever $\bar{g} / n_{\text {pixels }}>0.40$ (i.e. up to $1.6 \mathrm{kpc}$ above the plane). In the faintest region of the galaxy (above $1.6 \mathrm{kpc}$ from the plane), the main sources of error are large-scale flat-fielding errors and sky variations.

\subsection{Scattered Light?}

To see how much scattered light from the central part of the galaxy contributes at high-z, we constructed a stellar profile out to 150 pixels $(5.3 \mathrm{kpc})$. In the center, we used the stellar profile constructed using DAOphot (Section 3.1). For the outer region, we used two saturated stars. Using stars whose centers were saturated means that the signal-to-noise in the outer wings is higher than in unsaturated stars. We masked the stars in the wings of the saturated stars, as well as diffraction spikes around the saturated stars. The unmasked pixels were averaged in radial annuli. The profiles from the two saturated stars were averaged and joined to the DAOphot PSF profile. The combined profile was normalized to the surface brightness of the central bright region of NGC 4244. The final

profile is shown in Figure 6. At a distance of $2 \mathrm{kpc}$ ( 56 pixels) from galaxy center, the scattered light from the bulge is $\approx 28.5$ magnitudes $\operatorname{arcsec}^{-2}$, below our quoted photometric limit of $\mu_{R}=27.5$ magnitudes $\operatorname{arcsec}^{-2}$. Thus, it is not a significant effect.

\subsection{2-D Fits}

We fit a single exponential disk to the data:

$$
\begin{array}{r}
\rho_{\text {disk }}=L_{0} e^{-R / h_{R}} e^{-|z| / h_{z}} \quad\left(R<R_{\max }\right) ; \\
=0 \quad\left(R \geq R_{\text {max }} .\right)
\end{array}
$$


(here $L_{0}$ is the central density of the disk in $L_{\odot} \mathrm{pc}^{-3}$ ). The two-dimensional modeling is described fully in Morrison et al. (1994). We start with a full three-dimensional model, and then for each iteration of the fit, we numerically integrate along the line of sight and produce a two-dimensional model, which we compare with the observed surface brightness.

We start out with eight parameters: the scale length and scale height $h_{R}$ and $h_{Z}$, inclination $i$, the coordinates of the galaxy center $x_{C}$ and $y_{C}$, the radial extent of the galaxy $R_{\max }$, the central surface brightness $I_{C}$, and the sky value. Because of high correlations among the parameters, eight free parameters are extremely difficult to fit accurately, but, fortunately, some of the parameters can be fixed using other information. The sky value is determined by averaging the values in any bin with more than 100 pixels, away from the galaxy, as described in Section 3.2. The $x_{C}$ and $y_{C}$ position of the center of the galaxy and $R_{\max }$ are determined from a contour plot of the galaxy and by making one-dimensional profiles across the minor axis and parallel to the major axis. The inclination $i=84.5^{\circ}$ was adopted from Olling's (1996a) kinematic measurement. Only three parameters then need to be determined from the $2-\mathrm{D}$ fitting of a single exponential disk: $h_{R}, h_{Z}$, and central surface brightness.

How well do 2-D fits work? Because we are using a Levenberg-Marquardt algorithm (Press et al. 1992), which is an iterative nonlinear leastsquares algorithm, we need to provide starting values. We investigated the sensitivity of the algorithm to the initial estimates used by mapping out the value of $\chi^{2}$ over a large grid of starting values and noting where the algorithm converged. We find that if all three parameters, $h_{R}, h_{Z}$, and $I_{C}$, are estimated, then the initial estimates need to be within $20 \%$ of the correct values for the model fit to converge. If one of the three parameters can be estimated from other information, such as $h_{Z}$ from the 1-D fits, then initial estimates of the other two can be as much as $50 \%$ wrong and the model will still converge successfully. A starting grid of $\chi^{2}$ can be used as a "Levenberg-Marquardt-by-eye" to locate the "trench", that is, the starting parameter values for which the model fit will converge. Once the trench has been found, all three parameters are allowed to vary.

As illustration, in Figure 7, we show a contour plot of $\chi^{2}$ for fixed $h_{Z}=6.5$ pixels (233 pc). The contours are logarithmic; steep slopes are even steeper than they appear. For all starting values of $h_{R}$ and $I_{C}$ shown on the plot, except upper left $\left(h_{R} \approx 1000, I_{C} \approx 110\right)$, lower left $\left(h_{R} \approx 1000, I_{C} \approx 30\right)$, and lower right $\left(h_{R} \approx 3000, I_{C} \approx 30\right)$ corners, the starting model will converge. However, for $h_{Z}$ values which are more than $\approx 20 \%$ from the true $h_{Z}$, the initial values of $h_{R}$ and $I_{C}$ need to be within $\approx 50 \%$ of the true values.

Another concern is the reliability of the estimates once the algorithm has converged. Morrison et al. (1994) showed that estimates based on the covariance matrix of the fit 
can underestimate the true values severely. Thus, to determine error estimates on the derived disk parameters $h_{R}, h_{Z}$, and $I_{C}$, we used bootstrapping with resampling (Efron 1982, Chapter 15 of Press et al. 1992, Efron \& Tibshirani 1993). We ran our 2-D model fitting program 1000 times. Each time, the data to be fit were chosen by random sampling with replacement from the actual data. The sets of resulting disk parameters were roughly Gaussian distributions, centered on the result of the run using all of the data. The errors estimated from the widths of bootstrapping analysis are more reasonable estimates of our true errors, as the bootstrapping errors include not only the formal errors from known contributions but also a more realistic estimate of goodness of fit and systematics (unmasked foreground stars, dust, and small, faint, background galaxies). In Figure \&, we show a histogram of $h_{Z}$ values estimated by our bootstrapping code.

In Table 2, we show the results of our 2-D fitting with the bootstrapping errors, as well as the correlation coefficients between all the variables. To determine all of the correlation coefficients, all eight variables were started at their correct values and allowed to vary. The important correlation coefficients are those between the first three variables, the scale length $h_{R}$, the scale height $h_{Z}$, and the central surface brightness $\mu_{0}$ ? But the strong correlations between some of the other variables, such as between $h_{Z}$ and inclination, or $R_{\max }$ and $h_{R}$, demonstrate why it is important to use other methods to estimate some of the parameters.

We estimated $R_{\max }$ directly from the image to reduce the effects of these correlations. Unfortunately, the galaxy is not axisymmetric: $R_{\max }$ on the southwest side of NGC 4244 (left side of Figure 4) is $8 \%$ larger than on the northeast side: $10.5 \mathrm{kpc}$ vs $9.7 \mathrm{kpc}$. We chose an intermediate value of $10.0 \mathrm{kpc}$. Using the northeast side (right side of Figure 4) only in our estimation, with $R_{\max }=9.7 \mathrm{kpc}$, results in an estimate of $h_{R}$ which is $3 \%$ larger. The estimates of $h_{Z}$ and $\mu_{0}$ change an insignificant amount. The bright stars and H II regions at the left edge of NGC 4244 make it difficult to model the left side only.

\subsection{Vertical Profiles}

In Figure 9, we show vertical profiles parallel to the minor axis. Our best fit model of a single exponential disk with scale height of $246 \mathrm{pc}$ is shown with the data. A single disk fit is a very good match to the data down to 27.5 magnitude $\operatorname{arcsec}^{-2}$ and there is

\footnotetext{
${ }^{5}$ Note that the central surface brightness quoted here is for the observed inclination of $84 .{ }^{\circ} 5$. For comparison to other galaxies, the face-on and edge-on central surface brightnesses for this model are also quoted.
} 
little evidence for disk flaring, i.e. changing scale height as a function of radius. The only profile that appears to flare is the one at $\pm 8.5 \mathrm{kpc}$. However, the warp in NGC 4244 starts at $\approx 8.0 \mathrm{kpc}$ and we attribute the apparent flaring to the warp. In the galaxy center, we may have a small contribution to the light from the bulge, which may lead to a slight underestimate of $h_{Z}$ there, due to the steeper bulge profile.

To test the constancy of $h_{Z}$ with radius, we split the galaxy into inner and outer regions, $R<4 \mathrm{kpc}$ and $4<R<8 \mathrm{kpc}$ (the region outside $R=8 \mathrm{kpc}$ was not included to minimize the effect of the small warp), and analyzed the two regions separately. For the inner region, we find $h_{Z}=246 \pm 2 \mathrm{pc}$ and, for the outer region, we find $h_{Z}=259 \pm 8 \mathrm{pc}$. The difference between the inner and outer regions is not significant at the $2 \sigma$ level. This lack of evidence for flaring will be discussed further in Section 4.3.

In Figure 10 and 11, we show profiles parallel to the major axis, at $\pm 760 \mathrm{pc}$ and at \pm $955 \mathrm{pc}$ from the major axis. The horizontal profile at $\mathrm{z}= \pm 760 \mathrm{pc}$ is the closest distance to the major where a complete profile can be drawn. This is because of the large number of pixels masked due to dust, H II regions, and foreground stars at lower z. In Figure 10, the region further out than $\sim 7 \mathrm{kpc}$ from the minor axis shows more light than predicted by a single exponential disk fit, as if either the outer regions have a larger scale length $h_{R}$ or the inclination changes slightly with radius, becoming closer to edge-on for large $R$. Olling's (1996a) H I data also suggest that the inclination increases slightly with radius.

The profile at $\mathrm{z}= \pm 955 \mathrm{pc}$ shows the influence of the warp more clearly. Olling (1996a) found evidence for a warped H I disk. He found that the warp starts close to the edge of the optical disk ( $R=8.5 \mathrm{kpc}$ ), as is typical of galaxy warps (Briggs 1990). The H I warp extends below the major axis on the left side of the disk and above on the right side in our Figure 3. Olling (1998) finds that its line of nodes is close to the line of sight. In Figure 11, the warp is apparent for the entire outer half of the disk, starting at $5 \mathrm{kpc}$ from the minor axis, where data from the four quadrants of our image are quite different. In other words, in our faint optical data we see more details of the warped outer disk superimposed on the line of sight than is seen in the H I data, despite its high quality. (We do not show data past $7 \mathrm{kpc}$ on the left side because of the presence of a large region of current star formation and three bright stars.)

We also see evidence for a small warp in the outer parts of the stellar disk in our vertical profiles. The line of centers in the vertical profiles changes by $\approx 2.5$ pixels (89 pc) between the center and the left edge of the disk. We corrected for this effect before making Figure 9. This is equivalent to a change of $0 .^{\circ} 5$. The optical warp we find is in the same direction as the H I warp. The optical warp in NGC 4244 is much smaller than in NGC 5907 (Morrison et al. 1994) or NGC 4565, which permits accurate study of its outer stellar disk. 


\section{DISCUSSION}

\subsection{Comparison to Previous Surface Photometry}

van der Kruit \& Searle (1981a) used photographic photometry to obtain thin disk parameters for NGC 4244 and, recently, Olling (1996a) reanalyzed their photometry to rederive a disk scale height and disk scale length. We confirm the existence of a disk cutoff, first noted by van der Kruit \& Searle (1981a), with our deeper data. Kodaira \& Yamashita (1996) used $J$ and $K^{\prime}$ photometry to estimate thin disk parameters. We list our disk parameters with those of the other groups in Table 3, including the orientation ("as-observed", face-on, and edge-on). van der Kruit \& Searle (1981a) adopted a distance of $5 \mathrm{Mpc}$, rather than 3.6 Mpc used by the other studies, and, in Table 3, we have scaled their scale height and scale length to the distance adopted by the other studies. To compare their $B_{J}$ data to our $R$ photometry, we assume $B-B_{J}=0.2$ and $B-R=1.5$, colors typical for an old stellar population (Bottema 1989). van der Kruit \& Searle (1981a) fit their data to a sech ${ }^{2}$ model, rather than an exponential light distribution and we include an estimate of the central surface brightness from an exponential model: $\mu$ (exponential) $=\mu$ $\left(\operatorname{sech}^{2}\right)-0.75 \mathrm{mag}$.

IF we compare our $R$ photometry to van der Kruit \& Searle's $B_{J}$ photometry, we find a blue color $(B-R=0.2 \mathrm{mag}$, rather than $1.5 \mathrm{mag})$ for NGC 4244 . However, it is very difficult to determine accurate zero points from photographic photometry; $B$ CCD data are needed to determine the galaxy's color.

Our disk scale height is $15 \%$ larger than found by van der Kruit \& Searle (1981a) and by Olling (1996a), but our scale length is the same. Our disk parameters do not agree well with those found by Kodaira \& Yamashita (1996), however, their data are limited to the inner $180^{\prime \prime}$ (3.2 kpc), making the scale length more difficult to determine. In summary, our results agree well with previous optical studies.

In Table \&, we compare our disk parameters for NGC 4244 to those of NGC 5907 (Morrison et al. 1994) and the Milky Way (cf. Morrison et al. 1997). NGC 4244 has a smaller disk scale height than the other two galaxies and a fainter central surface brightness. The ratio of disk cutoff to radial scale length is similar to that of the Milky Way.

\subsection{Disk radial cutoff}

van der Kruit \& Searle (1981a) showed that stellar disks do not stretch out indefinitely, showing a sharp cutoff at $\sim 5$ radial scale lengths, well inside the H I disk. Our deep 
surface photometry of this almost unwarped stellar disk gives a strong test for disk cutoffs. Figure 12 shows profiles just inside $R_{\max }(<10 \mathrm{kpc})$ and outside it $(11.3 \mathrm{kpc})$. All the points outside $R_{\max }$ have $\mu_{R}$ fainter than 27 magnitude $\operatorname{arcsec}^{-2}$. The cutoff in stellar light happens relatively rapidly, over a distance of $1 \mathrm{kpc}$, and no faint outer component is visible down to $\mu_{R}=27.5$ magnitude $\operatorname{arcsec}^{-2}$. The existence of this stellar cutoff is consistent with the detection by Ferguson et al. (1998) of faint H II regions "at distance twice the optical radius" of the nearby face-on galaxies they studied. The "optical radius" (corresponding to a face-on surface brightness $\mu_{B}=25$ magnitude $\operatorname{arcsec}^{-2}$ ) is an outmoded concept based on what could be detected on photographic (POSSI) sky survey plates; it is now possible to see much fainter with CCD detectors. (For edge-on galaxies the situation is, of course, even easier.) Assuming $h_{R} / h_{Z}=10$ to convert face-on to edge-on surface brightness, $\mu_{B}=25$ corresponds to $\mu_{R} \sim 21$. Figure 9 shows that much of the stellar luminosity of NGC 4244 comes from outside the "optical radius" defined in this way.

\subsection{Constant Scale Height}

What determines the thickness of a stellar disk? Since its brightness varies exponentially with radius and the contribution of dark matter should not be strong in the inner galaxy, the weaker gravitational pull from the outer disk means that we would expect a component whose vertical energy does not vary with radius to flare towards the edges. This can be seen clearly for NGC 4244's H I in Fig. 13 of Olling (1996a), the scale height of H I increases from $\approx 400 \mathrm{pc}$ at a radius of $5 \mathrm{kpc}$ to $1.5 \mathrm{kpc}$ at $13 \mathrm{kpc}$. However, the factors which determine the scale height of a stellar disk are more complex: although stars are born out of ISM with low velocity dispersion which is constant with radius (Bronfman et al 1988, Wouterloot et al. 1990, Scoville et al. 1993, Combes \& Becquaert 1997), various heating mechanisms change the velocity dispersion and, thus, the scale height. The velocity dispersion of stars in the solar neighborhood increases with age (Jahreiss \& Wielen 1983).

This is thought to be due to heating by spiral arms and giant molecular clouds, with GMCs being particularly important for vertical heating (Jenkins \& Binney 1990). van der Kruit \& Searle found that the scale height of the stellar disks in the galaxies they studied (1981a,b, 1982a,b) remained constant with radius, despite the variation in CO surface density and, so presumably, numbers of GMCs with radius.

Recently, de Grijs \& Peltier (1997) claim evidence for disk flaring in their study of 48 edge-on galaxies, and for a trend of increasing flaring with Hubble type (in the sense that earlier types show more flaring). For S0 galaxies (de Vaucouleurs type $\approx-2$ ), they predict a change in scale height with radius of $\approx 9-10 \%$. For Sc galaxies (de Vaucouleurs type $\approx$ 
5 ), they predict a change in scale height with radius of $\approx 1-4 \%$, which is consistent with our result of a statistically insignificant variation between the inner and outer parts of the disk for NGC 4244. It is hard to understand this variation with Hubble type if the flaring is caused by the factors discussed above. de Grijs \& Peltier fit only regions between 1.5 and 4.0 scale lengths, so the gravitational effect of the bulge can be ignored in their study.

For earlier type galaxies, however, the determination of disk flaring is more difficult: as noted by de Grijs \& Peltier (1997), galaxy interactions, warps, and thick disks can also cause an apparent change in scale height. An incorrect bulge-disk decomposition will have the same effect. To distinguish flaring from the presence of a thick disk requires fainter surface photometry than a survey project like de Grijs \& Peltier's can achieve. Viewed from outside, the Milky Way's thick disk would be visible at $\mu_{I}=23$ magnitude $\operatorname{arcsec}^{-2}$ (Morrison 1999, assuming $R-I=0.5$ ), which is the surface brightness level at which de Grijs \& Peltier's single disk vertical profiles (their Figure 3) begin to deviate from a single exponential fit.

As discussed in Section 3.6, the adopted inclination, which can be an indication of a warp, can have a large effect on apparent flaring. Kinematic inclination determinations, such as Olling's for NGC 4244, do not exist for many galaxies, making the detection of some warps and correct modeling of the disks more difficult. de Grijs \& Peltier do not address the presence of warps in the galaxies they observed.

In conclusion, the question of how the thickness of stellar disks vary with radius is a thorny one to approach observationally, especially in early type galaxies where the existence of additional components like bulges and thick disks makes accurate separation of the thin disk light very difficult (see Morrison et al. 1997 for a discussion of how the problem is exacerbated if one-dimensional profiles are used instead of two-dimensional fits). Our data show a statistically insignificant increase in scale height between the inner and outer parts of the disk of NGC 4244, and it is possible that this is partially due to the small warp. This highlights the need for more theoretical work on disk heating mechanisms.

\subsection{Is there a second component?}

A single exponential disk (Equation 2) is a good fit to the data down to 27.5 magnitude $\operatorname{arcsec}^{-2}$ (see Figure 9). We do not see evidence of an extra component. However, our data may not rule out a faint component resembling the Milky Way's halo, which has a very centrally concentrated space distribution, with density $\rho$ varying as $r^{-3}$ or $r^{-3.5}$. NGC 4244 has a nucleus with total luminosity $2 \%$ of its disk's, which we calculate using the disk 
central surface brightness and bulge effective intensity given in Baggett et al. 1998, and a bulge effective radius of $4^{\prime \prime}(71 \mathrm{pc})$ 巳. What if this nucleus was the central part of such a halo? We assume that the core radius of this putative halo is two pixels $(71 \mathrm{pc})$, and calculate what brightness a power-law halo which fit smoothly onto the luminosity distribution of the nucleus would be. On the minor axis, $2 \mathrm{kpc}$ above the plane, the $r^{-3}$ halo would have a brightness $\mu_{R}=26.5$ magnitude $\operatorname{arcsec}^{-2}$, which our data rule out, but the $r^{-3.5}$ halo would have a brightness $\mu_{R}=28.4$ magnitude $\operatorname{arcsec}^{-2}$, below our detection limit.

It is interesting to compare NGC 4244 with its Local Group analog, M 33, which has a bulge-to-disk ratio of $\sim 0.02$ (Bothun 1992). While it is difficult to search for halo field stars in M 33 because of its nearly face-on inclination and badly warped disk, there is evidence for a small number of globular clusters with halo kinematics. Schommer et al. (1991)] show that M 33 has $\sim 20$ old clusters in a kinematically hot configuration (see also Sarajedini et al. 1998). Whether these clusters are better associated with M 33's disk or tiny bulge, their kinematics suggest that any field stars associated with them would have a larger scale height than the thin disk.

There have been no searches for globular clusters in NGC 4244, but we see no evidence for an extended field star population in this galaxy. A globular cluster search in NGC 4244 would help us decide whether it truly has no halo.

NGC 5907, a more luminous Sc, has a second component, which does not resemble either the Milky Way's halo or thick disk (Sackett et al. 1994, Morrison et al. 1994).

\section{CONCLUSIONS}

We have obtained deep $R$ band surface photometry of the small, edge-on Scd galaxy NGC 4244.

1. We find that NGC 4244 is well-described by a single exponential disk with a scale height of $246 \pm 2 \mathrm{pc}$, a scale length of $1.84 \pm 0.02 \mathrm{kpc}$, and a radial cut-off at 5.4 disk scale lengths. The thin disk parameters, $h_{R}, h_{Z}$, and $R_{\max }$ for NGC 4244 are smaller than those of NGC 5907 by about $50 \%$ (cf. Morrison et al. 1994).

\footnotetext{
${ }^{6}$ Baggett et al. give a bulge effective radius of $400^{\prime \prime}(7.1 \mathrm{kpc})$. We assumed that this was actually $4^{\prime \prime}(71$ $\mathrm{pc})$.

${ }^{7}$ the core radius of the Milky Way's halo is not well determined but is probably less than 500 pc based on counts of RR Lyraes in bulge fields
} 
2. NGC 4244 is a good galaxy for investigating stellar disk flaring because it does not have a significant thick disk and because it has no large companions. We find, in agreement with van der Kruit \& Searle (1981a), statistically insignificant flaring in the stellar disk. The warp in NGC 4244 may exaggerate the marginal disk flaring that we see.

3. We have placed stronger limits on the existence of a cutoff in the stellar disk.

4. Unlike the more luminous Sc galaxies M 33 and NGC 5907, NGC 4244 does not show a significant second component.

\section{A. The Error Model}

It is necessary to have accurate error estimates for both the 1-D and 2-D fitting. Unlike earlier photographic work, deep CCD surface photometry allows us to quantify measurement errors. Measurement errors arise from CCD behavior such as readout noise and flat-fielding, as well as from intrinsic galaxy variations. Each error contribution will be addressed below.

\section{A.1. Readout Noise}

The readout noise per exposure is 1.2 ADU. By combining 66 galaxy images with a median, we are able to reduce the effective readnoise to

$$
R_{e f f}=1.2 \mathrm{ADU} \frac{1.22}{\sqrt{\mathrm{N}_{\mathrm{G}}}}=0.18 \mathrm{ADU} .
$$

Averaging the 66 frames would have reduced $R_{\text {eff }}$ to 0.15 ADU; however, combining with a median is preferable for the removal of cosmic rays and other data defects. The factor of 1.22 is because of the reduced efficiency of a median (see Morrison, Boroson \& Harding 1994).

\section{A.2. Photon Noise}

For $C \mathrm{ADU}$ in a given pixel, the photon noise is $\sqrt{C / g}$, where $g$ is the gain. Combining 66 galaxy images using a median reduces the photon noise to

$$
\sigma_{\text {Poisson }}=\frac{1.22}{\sqrt{66}} \frac{\sqrt{C}}{\sqrt{g}} .
$$


In the final combined galaxy image, the contribution to the error from photon noise is $0.137 \sqrt{C}$ ADU.

\section{A.3. Flatfielding Errors}

In principle, the only limit to the precision of the combined flat field image is the photon noise in the individual flat field images. This small-scale variation is

$$
\sigma_{s f f}=\frac{\sqrt{C_{S}}}{\sqrt{g}} \frac{1.22}{\sqrt{N_{f}}} \frac{1.22}{\sqrt{N_{G}}}
$$

where $C_{S}$ is the number of counts in the final, combined master sky flat image, $g$ is the gain, $N_{F}$ is the number of individual sky flats used to make the master sky flat, and $N_{G}$ is the number of individual galaxy images used to make the final galaxy image. The sky counts $C_{S}=1998.0 \mathrm{ADU}$, the gain is $1.2 \mathrm{ADU}$, and the numbers of sky and galaxy images are 70 and 66 , respectively.

In practice, the small-scale flat fielding errors are not the only flat-fielding error we have. There are also large-scale variations which arise from the variation of the sky brightness across an image and from the wings of bright stars which have not been completely removed by combining the individual sky flats. To estimate the large scale variations $\sigma_{l f f}$, we divided our sky flats into two subsets of 12 each and constructed two mini-master sky flats. We then used IRAF's blkavg to bin the mini-masters into 100x100 pixel bins, to remove small-scale variations, and divided one mini-master into the other. The standard deviation of the divided images was $0.085 \% C_{S}$. The large-scale variation

$\sigma_{l f f}$ is reduced by $1 / \sqrt{2}$ because the mini-masters were constructed from half as many sky images as the master and by another $1 / 2$ because of the division of the two mini-masters. The large scale flat-fielding variation $\sigma_{l f f}=0.03 \% C_{S}$.

\section{A.4. Surface Brightness Fluctuations}

Another source of error arises from intrinsic variations in the galaxy. The light in each pixel comes from individual stars, and, thus, is subject to counting statistics. The variance of the brightness fluctuations is characterized by Tonry \& Schneider (1988). Following their Equation 10 and Morrison et al. (1994), we used $t=600 s, d=3.6 \mathrm{Mpc}, \bar{M}=0$ (for the $R$ band), and $m_{1}=22.23$ magnitude $\operatorname{arcsec}^{-2}$. The variance of the brightness is $3.61 \bar{g}$, where $\bar{g}$ is the number of counts from the galaxy only. 
Seeing has the effect of lessening the apparent brightness fluctuations. We modeled the effect of seeing empirically, by creating an IRAF image with known $\sigma_{\text {orig }}$ and smoothing the image by a Gaussian of similar FWHM as our typical seeing. The smoothed image was binned in bins of $3 \times 3$ to $50 \times 50$ pixels and $\sigma$ recalculated for each bin size. Without the effect of seeing, $\sigma_{\text {orig }}$ would be reduced by a factor of $1 / \sqrt{n}$, where $n$ is the bin size. We calculated the residuals $\Delta \sigma=\sigma-\sigma_{\text {orig }} / \sqrt{n}$ for each bin size. A function was fit to the $\Delta \sigma$ residuals and this function was used to correct the surface brightness variation errors for seeing.

\section{A.5. Sky Variations}

Our error in determining the sky level was $0.8 \mathrm{ADU}$, or $R=29.3$ magnitude $\operatorname{arcsec}^{-2}$, obtained from the histogram shown in Figure 5 .

\section{REFERENCES}

Aaronson, M., Bothun, G., \& Mould, J. R. 1986, ApJ, 302, 536

Baggett, W. E., Baggett, S. M., \& Anderson, K. S. J. 1998, AJ, 116, 1626

Binggeli, B., Sandage, A., \& Tammann, G. A. 1988, ARA\&A, 26, 509

Bothun, G. D. 1992, AJ, 103, 104

Bottema, R. 1989, A\&A, 221, 236

Briggs, F. H. 1990, ApJ, 352, 15

Bronfman, L., Cohen, R. S., Alvarez, H., May, J., \& Thaddeus, P. 1988, ApJ, 324, 248

Combes, F. \& Becquaert, J.-F. 1997, A\&A, 326, 554

Davis, M., Efstathiou, G., Frenk, C. S., White, S. D. M. 1985, ApJ, 292, 371

de Grijs, R. \& Peltier, R. F. 1997, A\&A, 320, L21

de Vaucouleurs, G. 1975, ApJ, 202, 610

Dressler, A. 1980, ApJ, 236, 351 
Edvardsson, B., Andersen, J., Gustafsson, B., Lambert, D.L., Nissen, P.E., Tomkin, J. 1993, A\&A, 102, 603

Efron, B. 1982, The Jackknife, the Bootstrap, and Other Resampling Plans, CBMS-NSF Regional Conference Series in Applied Mathematics, vol 38 (Society for Industrial and Applied Mathematics: Philadelphia, PA)

Efron, B. \& Tibshirani, R. J. 1993, An Introduction to the Bootstrap, Monographs on Statistics and Applied Probability 57 (Chapman \& Hall: New York, NY)

Eggen, O. J., Lynden-Bell, D., \& Sandage, A. R. 1962, ApJ, 136, 748

Freeman, K. C. 1970, ApJ, 160, 811

Ferguson, A. M. N., Wyse, R. F. G., Gallagher, J. S.,\& Hunter, D. A. 1998, ApJ, 506, 19

Jahreiss, H. \& Wielen, R. 1983 in "Nearby Stars \& Stellar Luminosity Function", IAU Colloquium 76, ed. A. G. D. Philip, A. G. Upgren (L. Davis Press: Schenectady, NY), 277

Jenkins, A. \& Binney, J. 1990, MNRAS, 245, 305

Kodaira, K., \& Yamashita, T. 1996, PASJ, 48, 581

Kraan-Kortweg, R. C. \& Tammann, G. A. 1979, AN, 300, 181

Landolt, A. U. 1992, AJ, 104, 372

Monkiewicz, J. A., Morrison, H. L., Harding, P., Boroson, T. A. 1999, in preparation

Morrison, H. L., Boroson, T. A., \& Harding, P. 1994, AJ, 108, 1191

Morrison, H. L., Miller, E. D., Harding, P., Stinebring, D. R., \& Boroson, T. A. 1997, AJ, 113,2061

Morrison, H. L. 1999, to appear in "The Third Stromlo Symposium: The Galactic Halo," ed. B. K. Gibson, T. S. Axelrod, \& M. E. Putnam, ASP Conference Series

Olling, R. 1996, AJ, 112, 457

Olling, R. 1996, AJ, 112, 481

Olling, R. 1998, private communication 
Press, W. H., Teukolsky, S. A., Vetterling, W. T., \& Flannery, B. P. 1992, Numerical Recipes in Fortran, Second Edition (Cambridge University Press, Cambridge)

Roberts, M. S. \& Haynes, M. P. 1994, ARA\&A, 32, 115

Quinn, P. J. \& Goodman, J. 1986, ApJ, 309, 472

Sackett, P. D., Morrison, H. L., Harding, P., Boroson, T. A. 1994, Nature, 370, 441

Sage, L. J. 1993, A\&A, 272, 123

Sarajedini, A., Geisler, D., Harding, P., \& Schommer, R. 1998, ApJ, 508, L37

Schommer, R. A., Christian, C. A., Caldwell, N., Bothun, G. D., \& Huchra, J. 1991, AJ, 101,873

Scoville, N. Z., Thakkar, D., Carlstrom, J. E., \& Sargent, A. I. 1993, ApJ, 404, 59

Searle, L. \& Zinn, R. 1978, ApJ, 225, 357

Silva, D. R., Boroson, T. A., Thompson, I. B., \& Jedrzejewski, R. I. 1989, AJ, 98, 131

Steidel, C. C., Giavalisco, M., Pettini, M., Dickinson, M., \& Adelberger, K. L. 1996, ApJ, 462, L17

Stetson, P. B. 1987, PASP, 99, 191

Tonry, J., \& Schneider, D. P. 1988, AJ, 96, 807

Tsikoudi, V. 1980, ApJS, 43, 365

Tully, R. B. 1988, Nearby Galaxies Catalog (Cambridge University Press: Cambridge, England)

van der Kruit, P. C., \& Searle, L. 1981a, A\&A, 95, 105

van der Kruit, P. C., \& Searle, L. 1981b, A\&A, 95, 116

van der Kruit, P. C., \& Searle, L. 1982a, A\&A, 110, 61

van der Kruit, P. C., \& Searle, L. 1982b, A\&A, 110, 79

Wolfe, A. M. 1990, in "The Interstellar Medium in Galaxies; Proceedings of the 2nd Teton Conference," ed. H. A. Thronson, Jr. \& J. M. Shull (Kluwer Academic Publishers: Dordrecht, Netherlands), 387 
Wouterloot, J. G. A., Brand, J., Burton, W. B., \& Kwee, K. K. 1990, A\&A, 230, 21 


\section{Figure Captions}

Fig. 1.- The environment of NGC 4244: galaxies in the B 4 and B 5 galaxy groups, plotted in supergalactic coordinates, from Tully 1988. Galaxies brighter than $M_{B, t o t}=-17.5$ (galaxies which are at least as bright as NGC 4244) are shown by solid symbols, less luminous galaxies by open symbols. Total range in luminosity is from $M_{B, t o t}=-13.0$ to -19.4 . We have circled NGC 4244.

Fig. 2.- Our final galaxy image, formed from 70 10-minute exposures. The orientation is $\mathrm{N}$ is up, $\mathrm{E}$ is to the left. The field shown here is $35^{\prime}$ by $30^{\prime}$.

Fig. 3. - Residuals from our photometric calibration are plotted as a function of $V-R$ color of the standard star. Since we observed in only one filter $(R)$, we are unable to correct for a color term in our calibration, but the plot shows that the effect of neglecting the color term is less than 0.10 mag over the whole range of stellar colors.

Fig. 4.- The galaxy image, rotated counter-clockwise by $42^{\circ}$ so that the major axis is parallel to the $\mathrm{x}$ axis, with our mask superimposed. The left side of the figure is the southwestern side and the right is the northeastern side.

Fig. 5.- Histogram of sky values from 310 large bins, located away from the galaxy.

Fig. 6. - The stellar PSF, normalized to have the same flux as the central region of NGC 4244. At a radius of $2 \mathrm{kpc}$ (56 pixels) the scattered light from the center of the galaxy contributes 28.5 magnitudes $\operatorname{arcsec}^{-2}$.

Fig. 7.- An example of a contour plot of starting $\chi^{2}$ values for fixed $h_{Z}=6.6$ pixels $(233$ pc). Contours are logarithmically spaced, with an interval of 0.1 . The final estimate for $h_{R}$ and $I_{C}$ is shown with a cross.

Fig. 8. - Histogram of $h_{Z}$ values estimated by our bootstrap code. The $\sigma$ measured from these data gives our error estimate for $h_{Z}$.

Fig. 9.- 1-D vertical profiles of NGC 4244, with our best fit exponential disk model shown as a solid line. The mean sky brightness of $R=20.9$ is marked with an arrow. Adjacent profiles have been shifted by $1.4 \mathrm{kpc}$ for clarity. Open circles are used for data to the right of the minor axis, filled for data to the left.

Fig. 10.- 1-D horizontal profile of NGC 4244, at \pm 760 pc from the major axis. Points to the left of the minor axis on Figure 4 are shown by open circles and points to the right of the minor axis are shown by closed circles. The point at $9.5 \mathrm{kpc}$ appears lower than it should, 
because it straddles the edge of the galaxy and so includes a section outside the disk cutoff.

Fig. 11. - 1-D horizontal profile of NGC 4244, at $\pm 955 \mathrm{pc}$ from the major axis. All four quadrants of Figure 4 are plotted using different symbols: closed circles and triangles are left of the major axis, open to the right, triangles below the major axis and circles above. The warp is clearly visible by $\sim 5 \mathrm{kpc}$ from the minor axis. The full line is the model.

Fig. 12.-1-D vertical profiles of the outer disk, showing the disk cutoff at $R_{\max }=10 \mathrm{kpc}$. The filled points lie within $R_{\max }$ and the open points outside of $R_{\max }$. By $9.9 \mathrm{kpc}$, the warp has a large enough effect that points at $9.9 \mathrm{kpc}$ do not lie on the model line. Open circles are used for data to the right of the minor axis, filled for data to the left. 
Table 1. Errors in a bin with 500 ADU from galaxy \& 1998 ADU from sky

\begin{tabular}{lcc}
\hline \hline \multicolumn{1}{c}{ Source } & ADU & $\%$ \\
\hline readout noise & 0.03 & 0.00 \\
Poisson stats & 0.75 & 0.03 \\
small-scale flat fielding & 0.10 & 0.00 \\
large-scale flat fielding & 0.60 & 0.02 \\
$\begin{array}{c}\text { surface brightness } \\
\quad \text { fluctuations }\end{array}$ & 4.60 & 0.19 \\
$\begin{array}{c}\text { large scale sky variations } \\
\quad+\text { sky determination }\end{array}$ & 0.80 & 0.03 \\
\hline
\end{tabular}

Table 2. Results of the 2-D Fitting. Parameters with errors quoted were estimated, the rest fixed.

\begin{tabular}{|c|c|c|c|c|c|c|}
\hline \multirow[b]{2}{*}{ Parameter } & \multirow[b]{2}{*}{ Value } & \multicolumn{5}{|c|}{ Correlation coefficients } \\
\hline & & $h_{R}$ & $h_{Z}$ & $\mu_{0}$ & inc. & $R_{\max }$ \\
\hline$h_{R}$ & $1.84 \pm 0.02 \mathrm{kpc}$ & 1.000 & 0.442 & -0.678 & -0.338 & -0.465 \\
\hline$h_{Z}$ & $246 \pm 2 \mathrm{pc}$ & & 1.000 & -0.512 & -0.520 & -0.110 \\
\hline$\mu_{0}$ (observed) & $20.47 \pm 0.05 R$ magnitudes $\operatorname{arcsec}^{-2}$ & & & 1.000 & -0.228 & 0.306 \\
\hline inc. & $84 .^{\circ} 5$ & & & & 1.000 & -0.039 \\
\hline$R_{\max }$ & $10.0 \mathrm{kpc}$ & & & & & 1.000 \\
\hline$\mu_{0}($ face-on $)$ & $22.07 R$ magnitudes $\operatorname{arcsec}^{-2}$ & & & & & \\
\hline$\mu_{0}$ (edge-on) & $19.90 R$ magnitudes $\operatorname{arcsec}^{-2}$ & & & & & \\
\hline
\end{tabular}


Table 3. Comparison to Previous Studies

\begin{tabular}{|c|c|c|c|c|}
\hline Author & $h_{Z}(\mathrm{pc})$ & $h_{R}(\mathrm{kpc})$ & $R_{\max }(\mathrm{kpc})$ & $\mu_{0}\left(\right.$ magnitude $\left.\operatorname{arcsec}^{-2}\right)$ \\
\hline van der Kruit \& Searle $(1981 a)^{a}$ & 209 & 1.87 & 9.91 & $\begin{array}{l}20.6\left(B_{J}, \text { edge-on, } \text { sech }^{2}\right) \\
\approx 19.9\left(B_{J}, \text { edge-on, exponen }\right. \\
\approx 20.1(B, \text { edge-on }) \\
\approx 18.6(R, \text { edge-on })\end{array}$ \\
\hline Kodaira \& Yamashita (1996) & 158 & 3.15 & - & - \\
\hline $\begin{array}{l}\text { Olling's (1996) reanalysis } \\
\text { of van der Kruit \& Searle (1981) }\end{array}$ & 210 & 2 & 10 & $\begin{array}{l}21.6 \pm 0.07(B, \text { face-on }) \\
\approx 17.6 \pm 0.07(R, \text { edge-on })\end{array}$ \\
\hline This study & $246 \pm 2$ & $1.84 \pm 0.02$ & 10.0 & $\begin{array}{l}20.47 \pm 0.05(R, \text { observed }) \\
19.90 \text { ( } R, \text { edge-on }) \\
22.07 \text { ( } R, \text { face-on })\end{array}$ \\
\hline
\end{tabular}

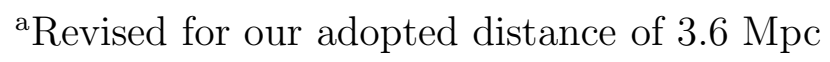

Table 4. Comparison of Thin Disk Parameters between NGC 4244, NGC 5907, \& the Milky Way

\begin{tabular}{lcc}
\hline \hline \multicolumn{1}{c}{ Galaxy } & $h_{Z}(\mathrm{pc})$ & $R_{\text {max }} / h_{R}$ \\
\hline NGC 4244 & 246 & 5.4 \\
NGC 5907 & 470 & 3.8 \\
Milky Way $^{\mathrm{a}}$ & 300 & 5.7 \\
\hline
\end{tabular}

${ }^{\text {a }}$ References for Milky Way values can be found in Morrison et al. (1997) 


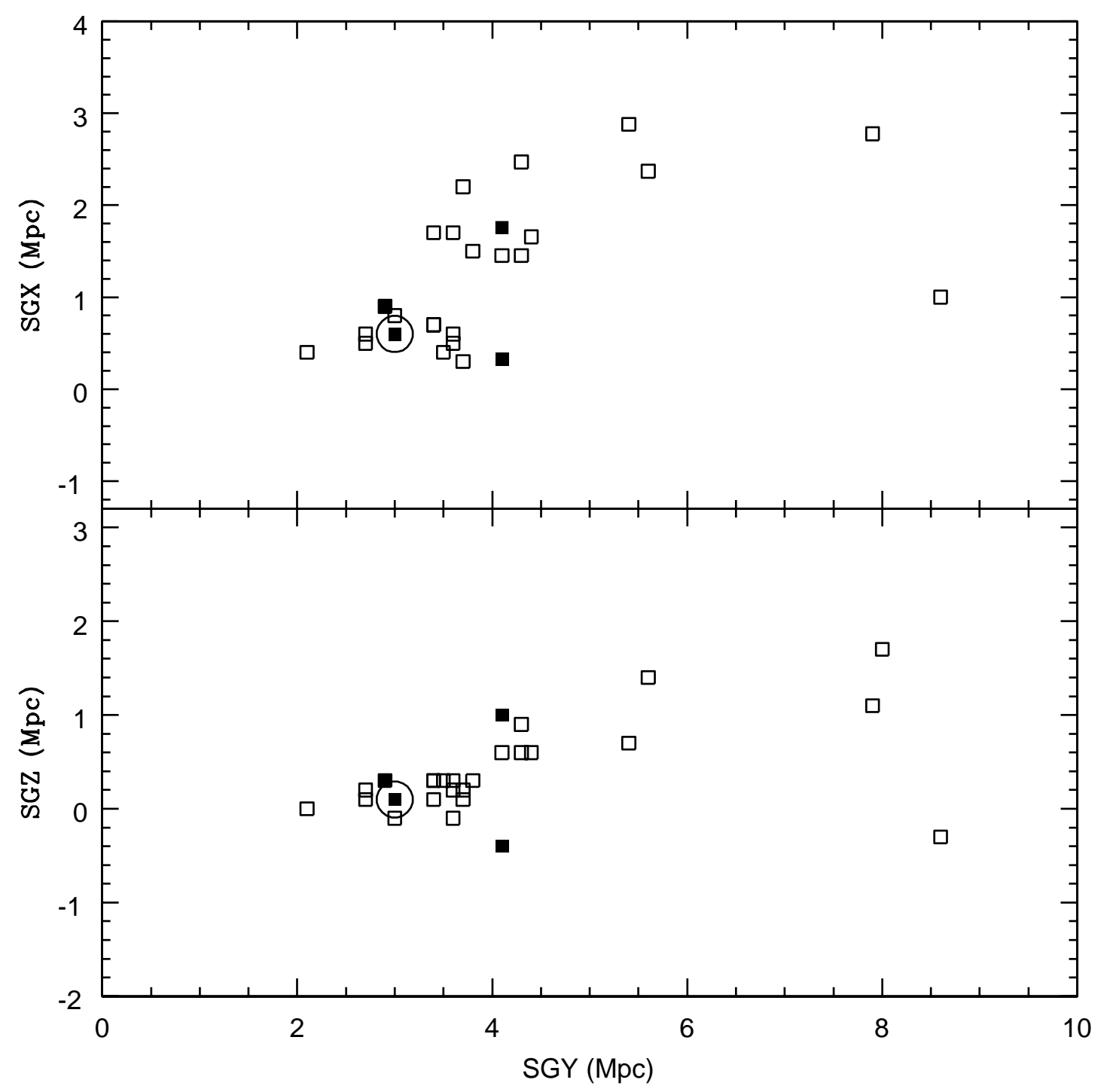




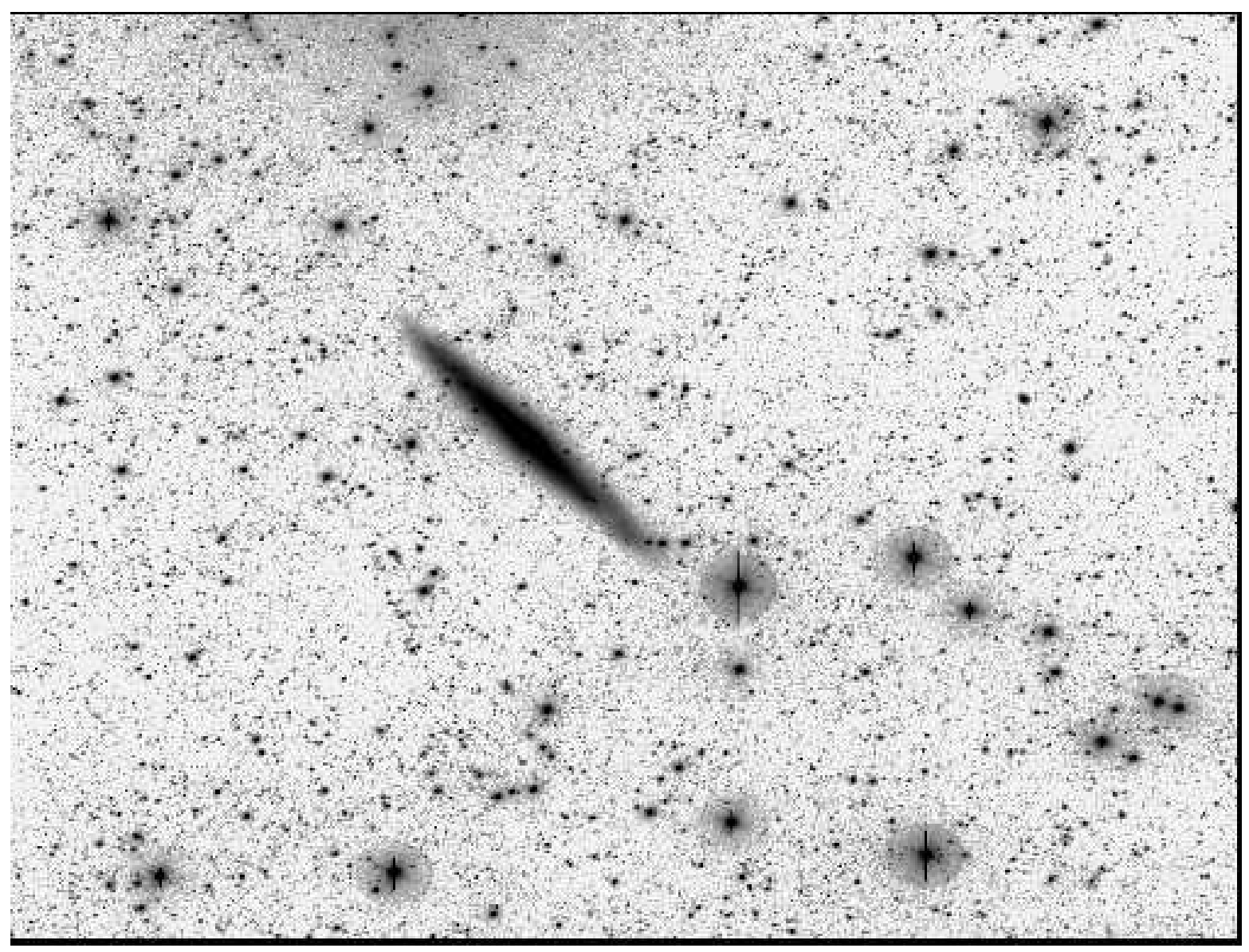




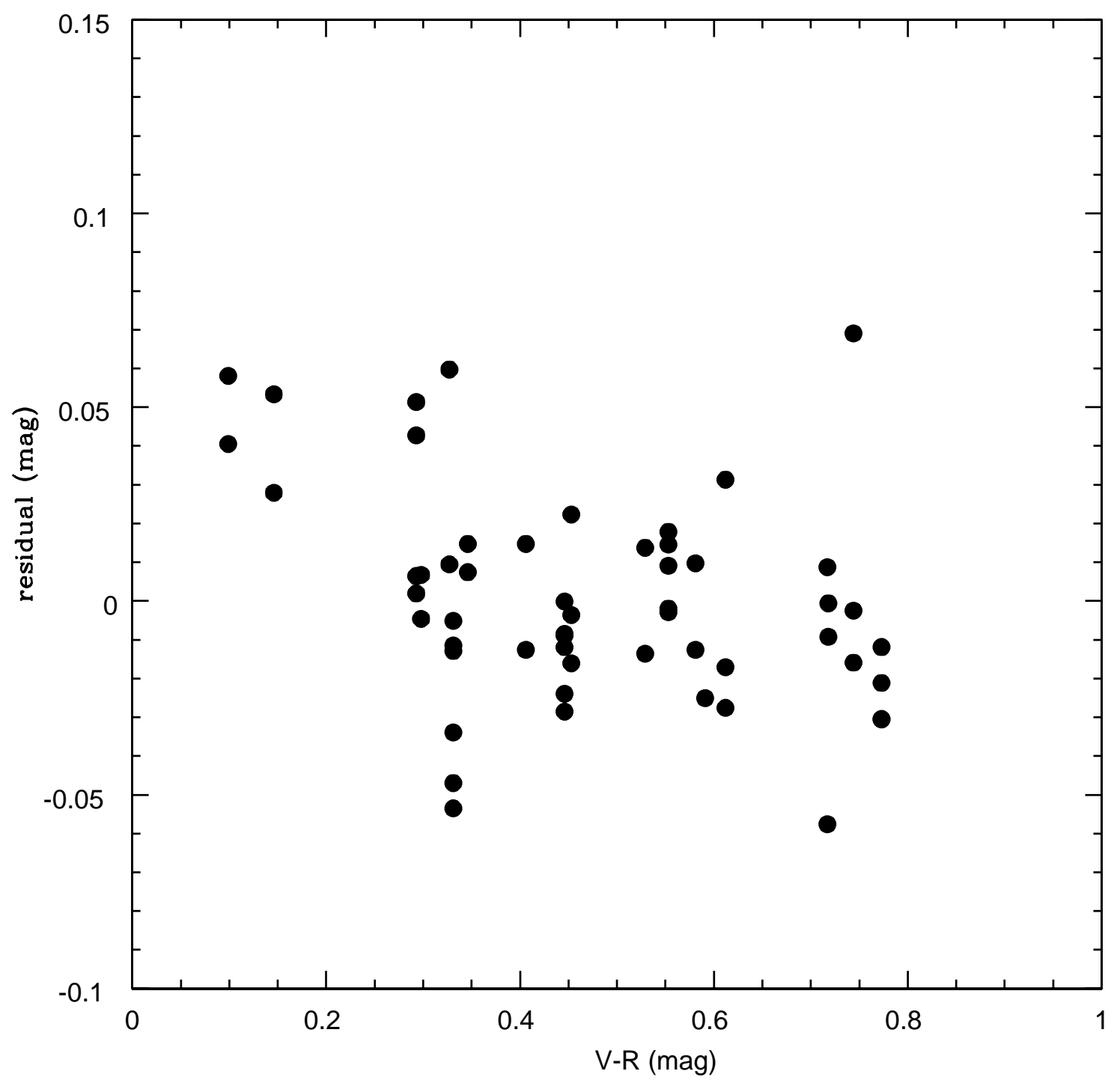




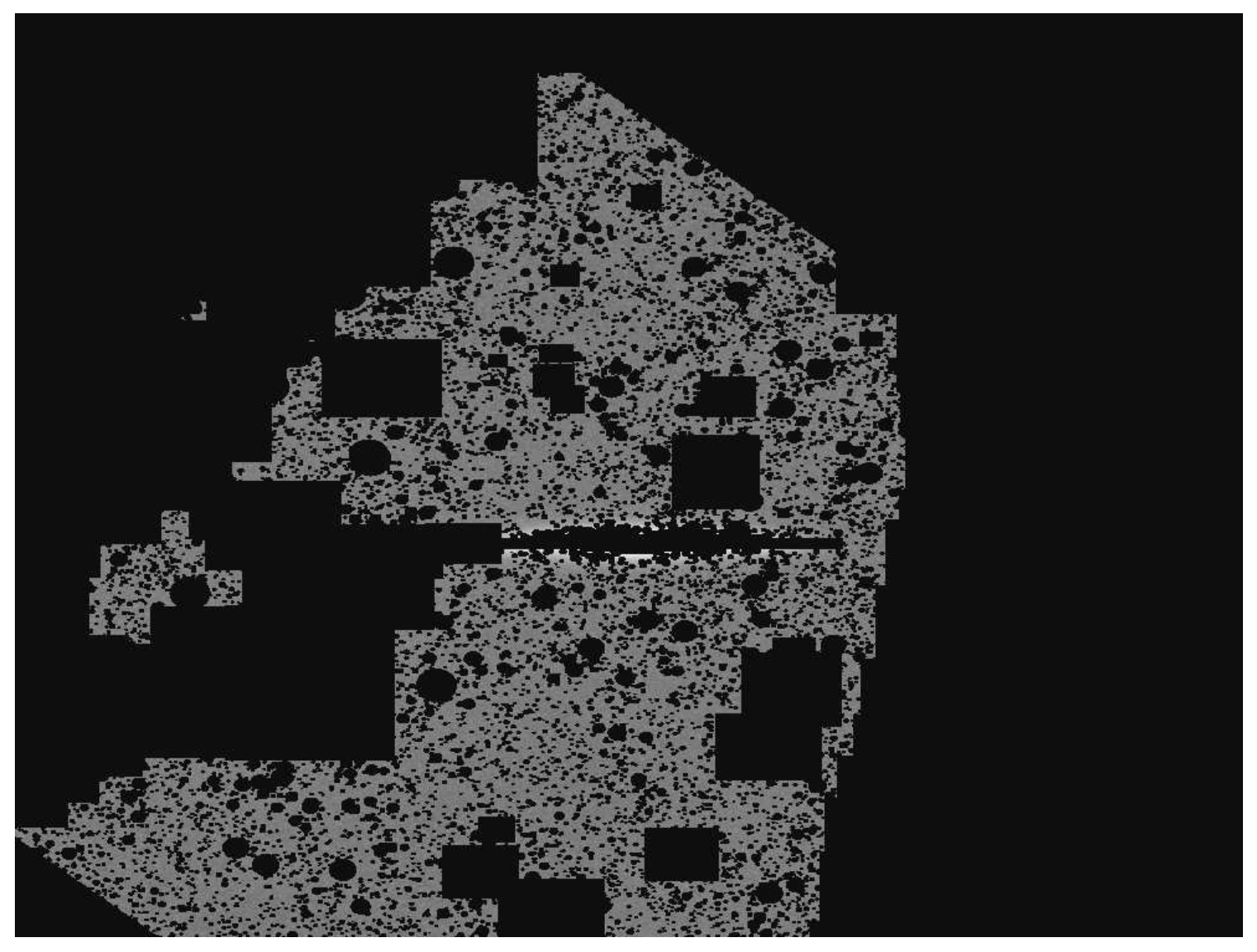




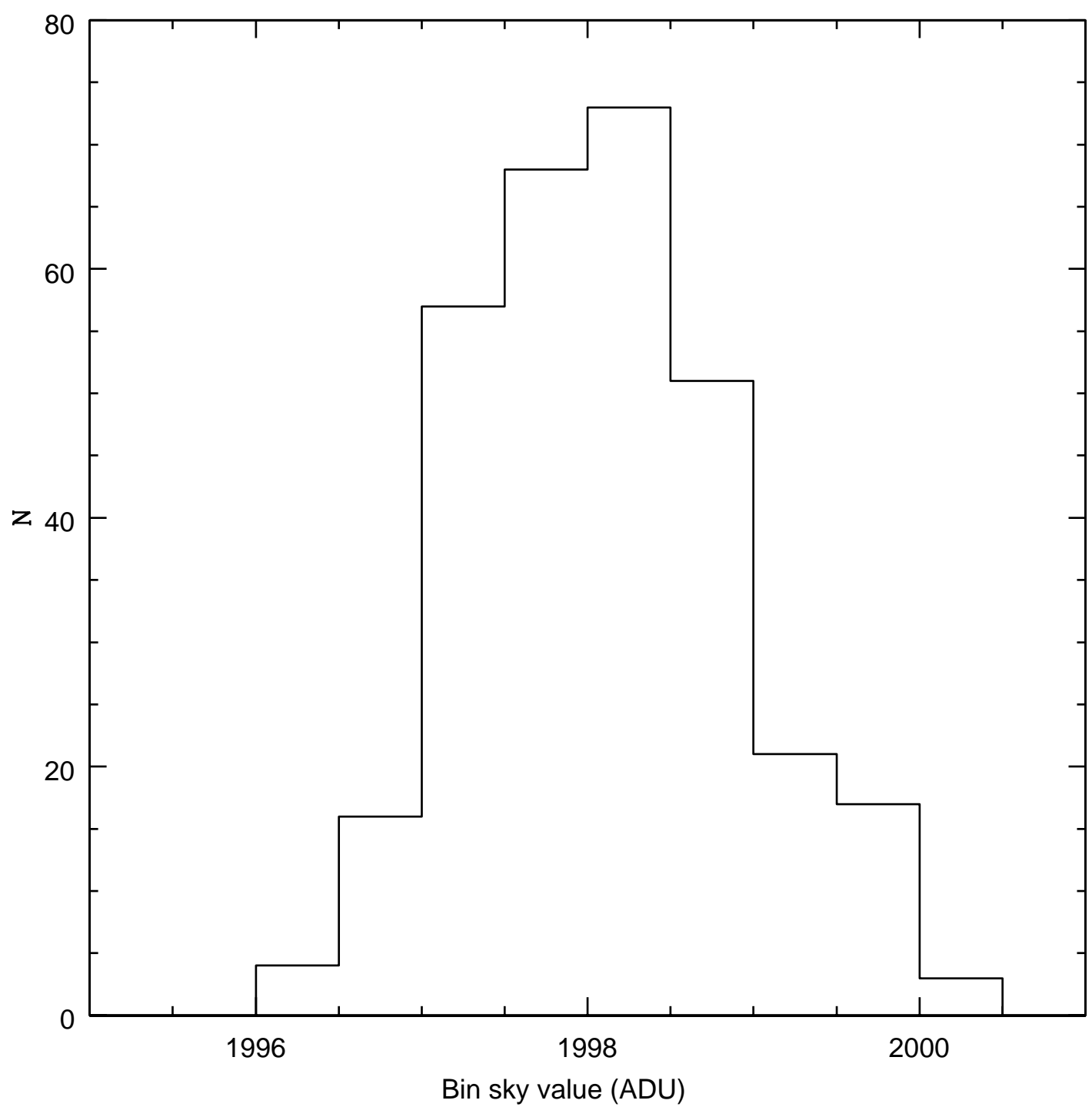




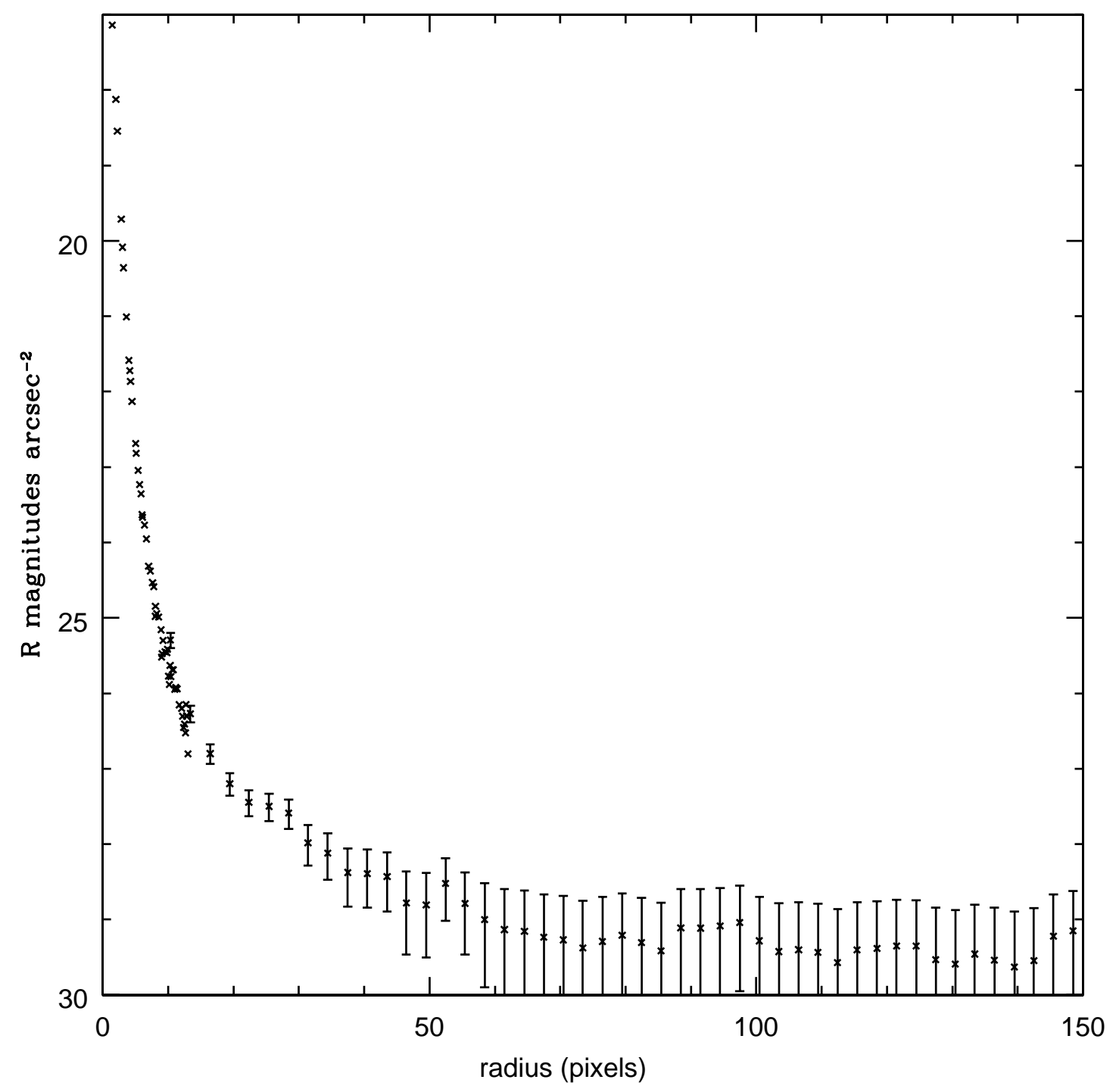




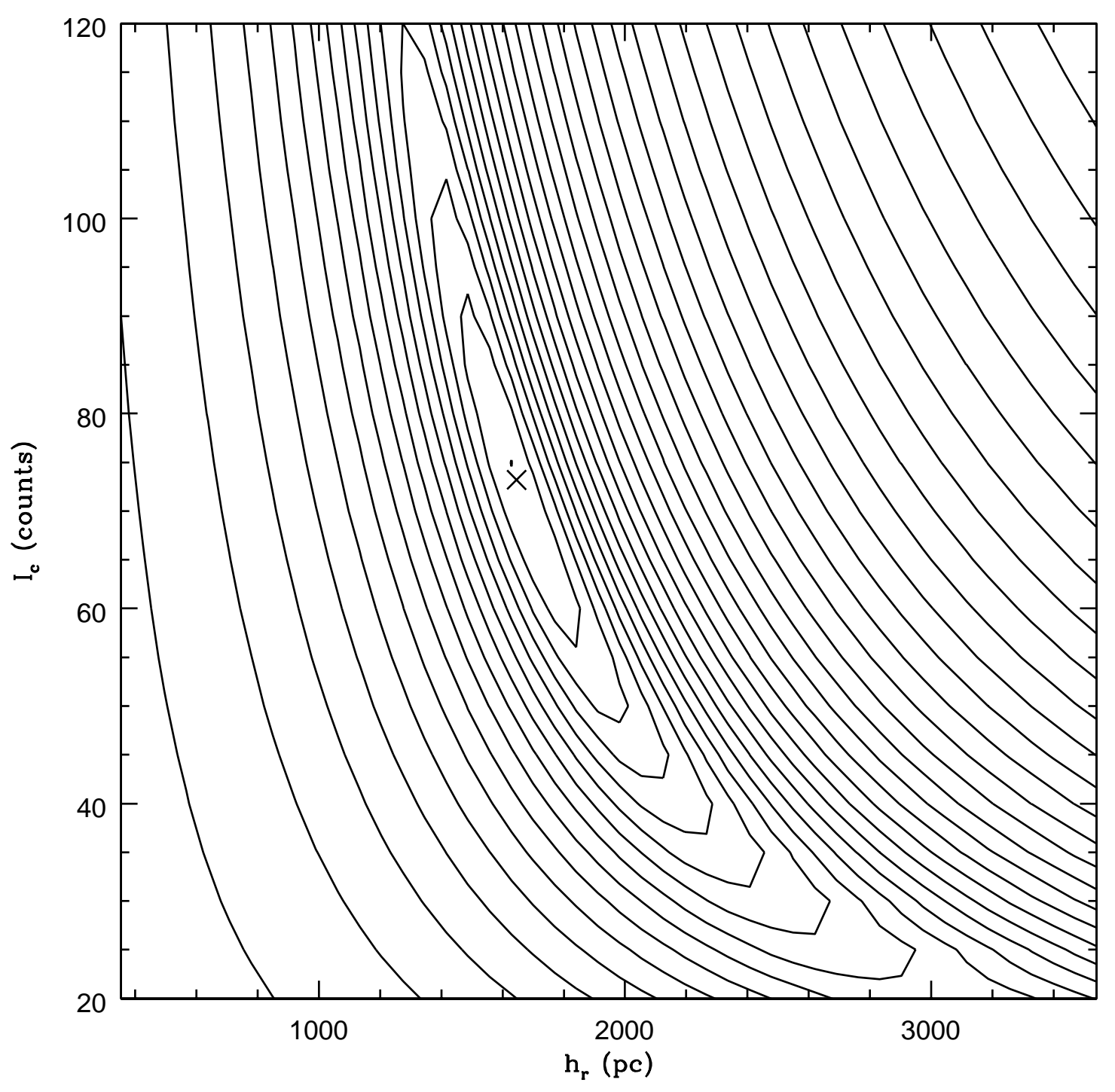




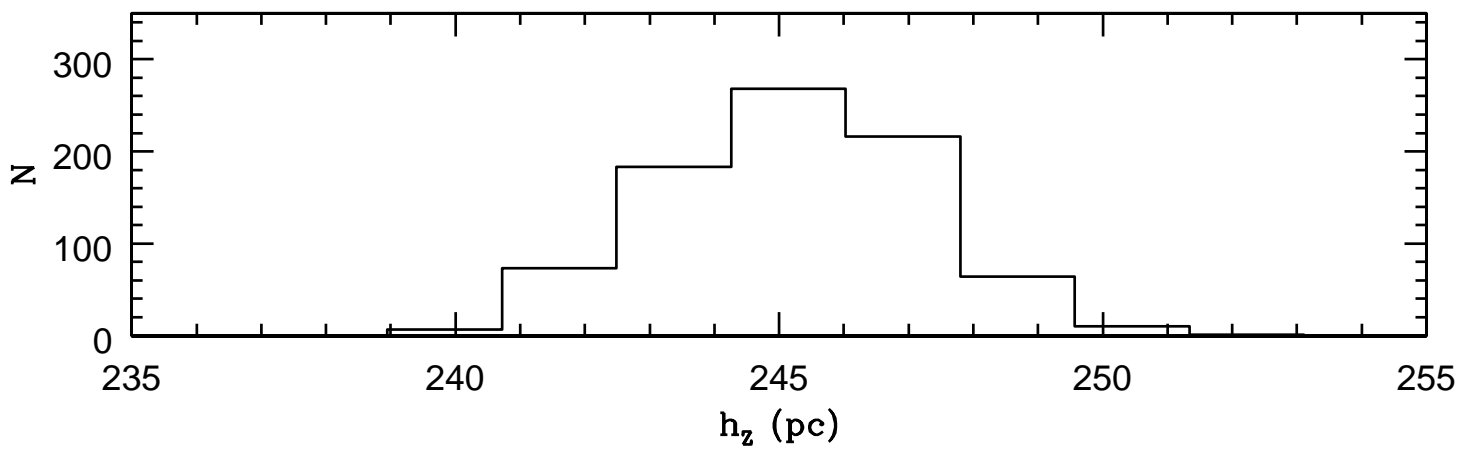




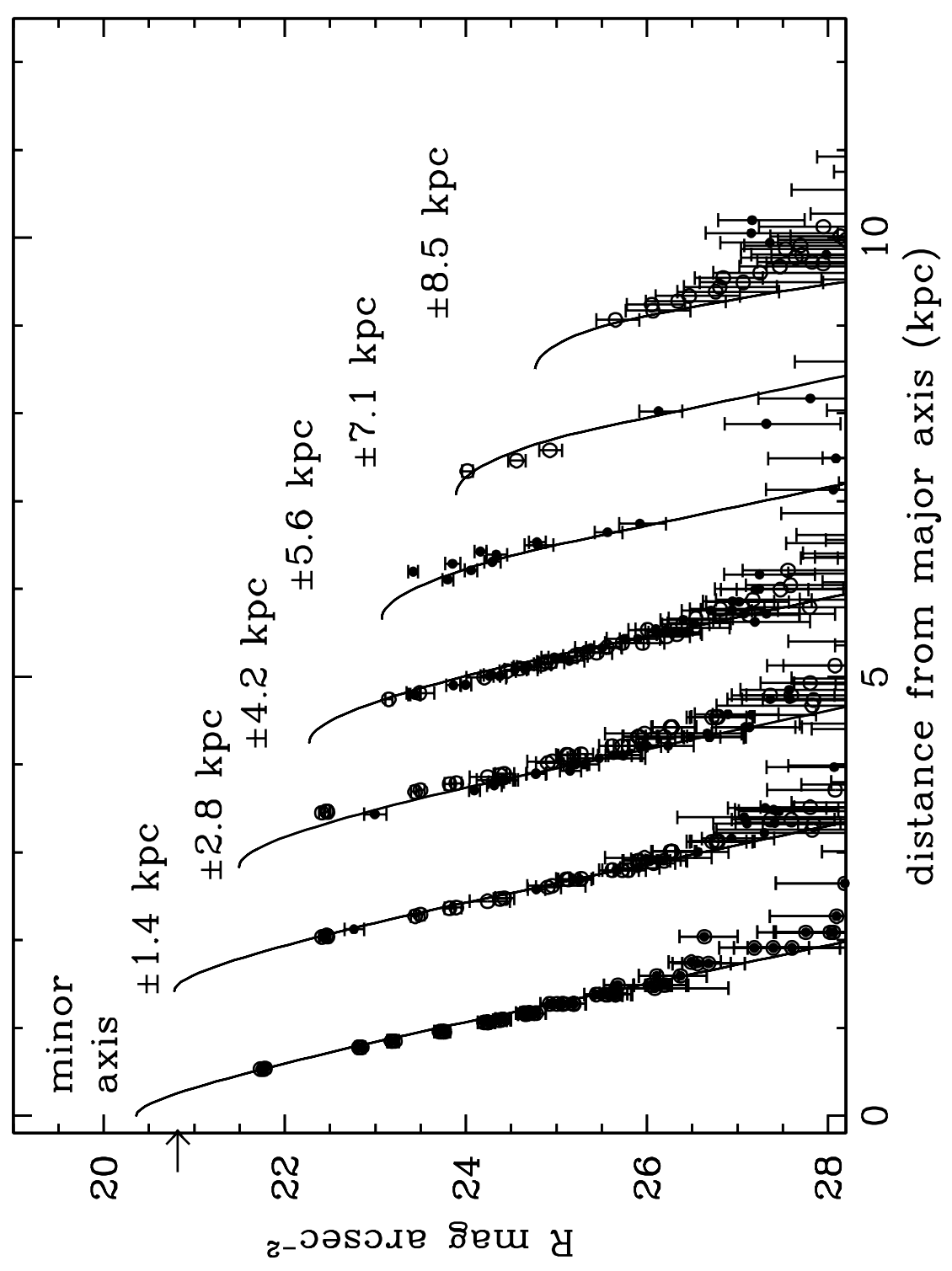




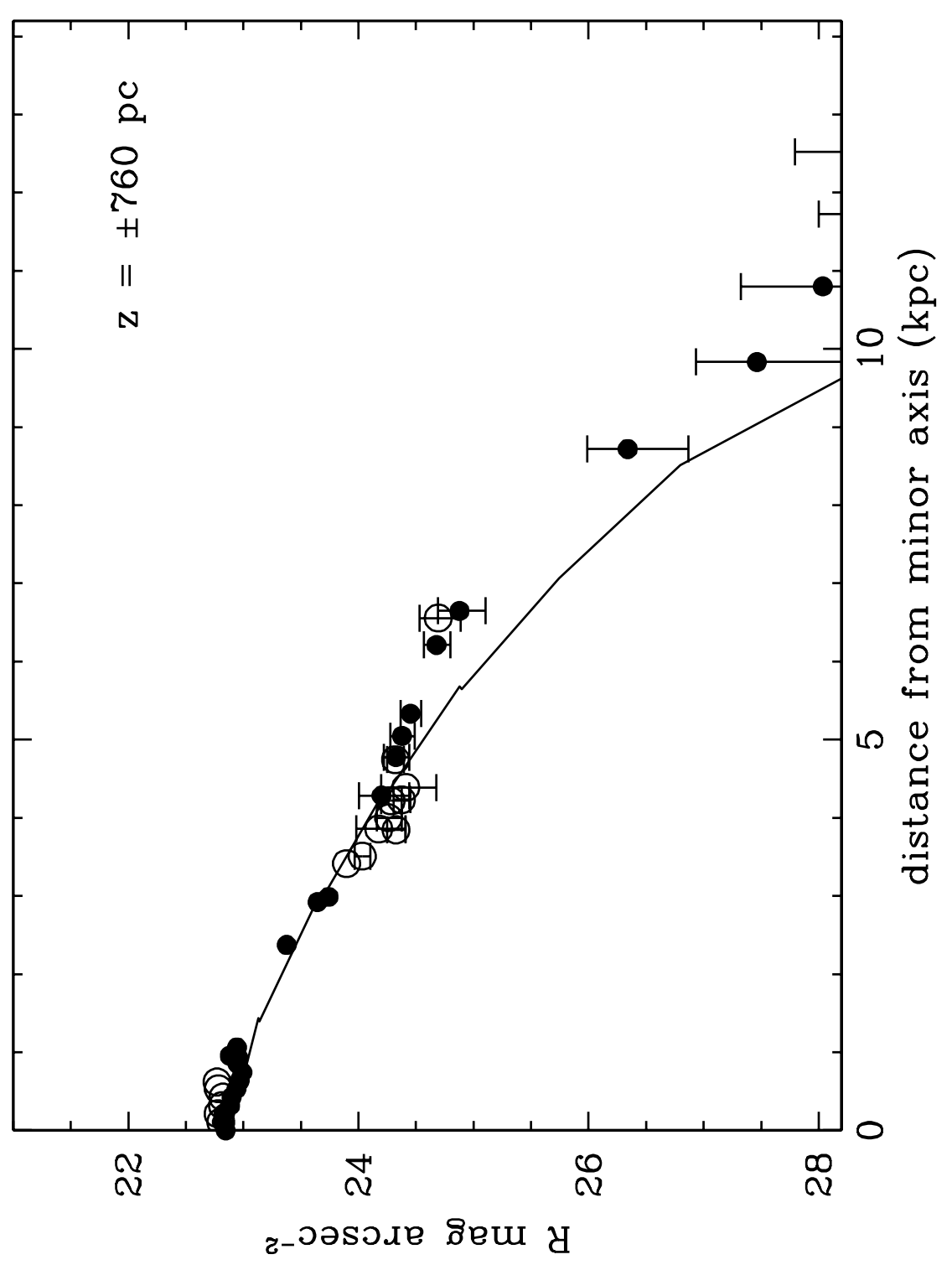




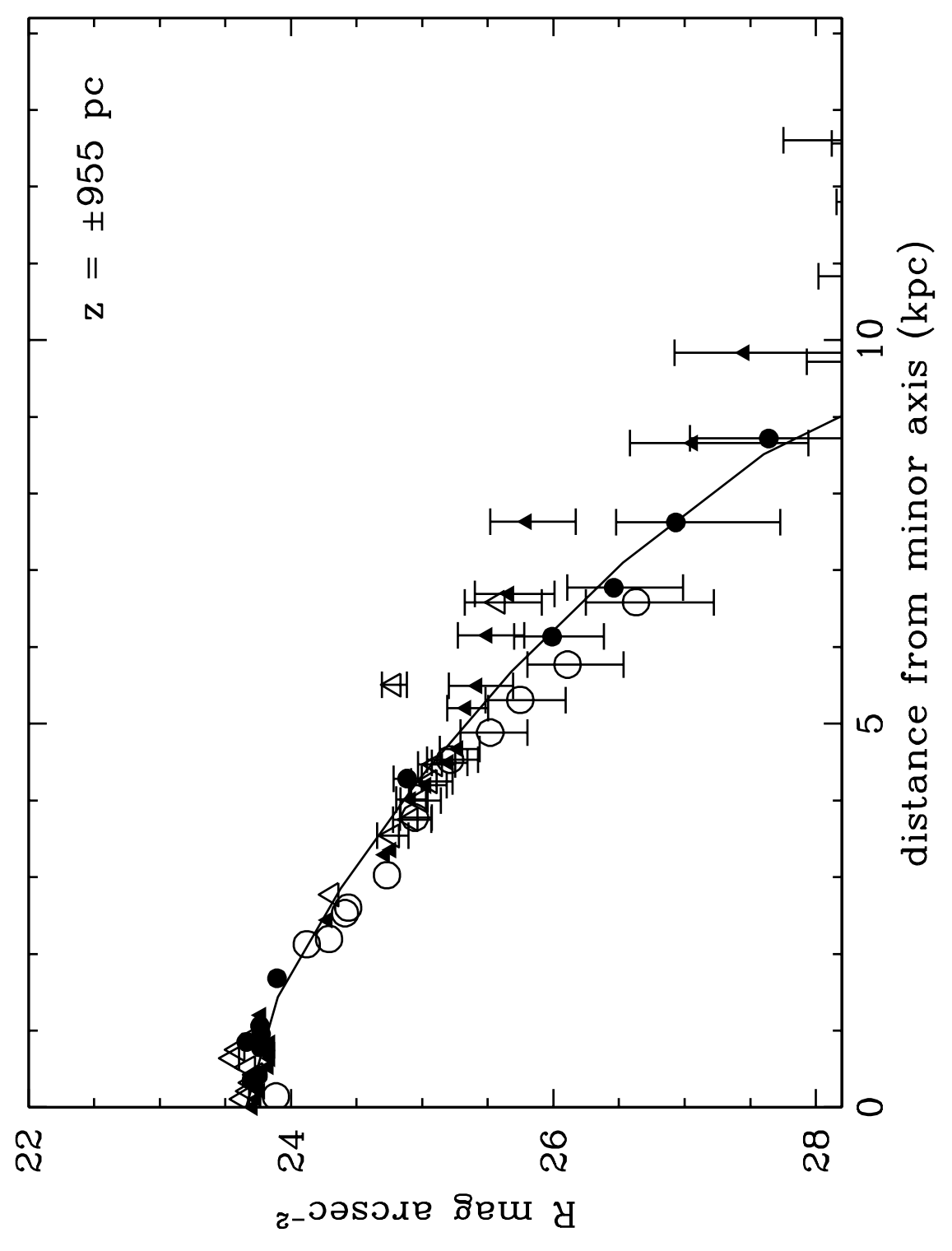




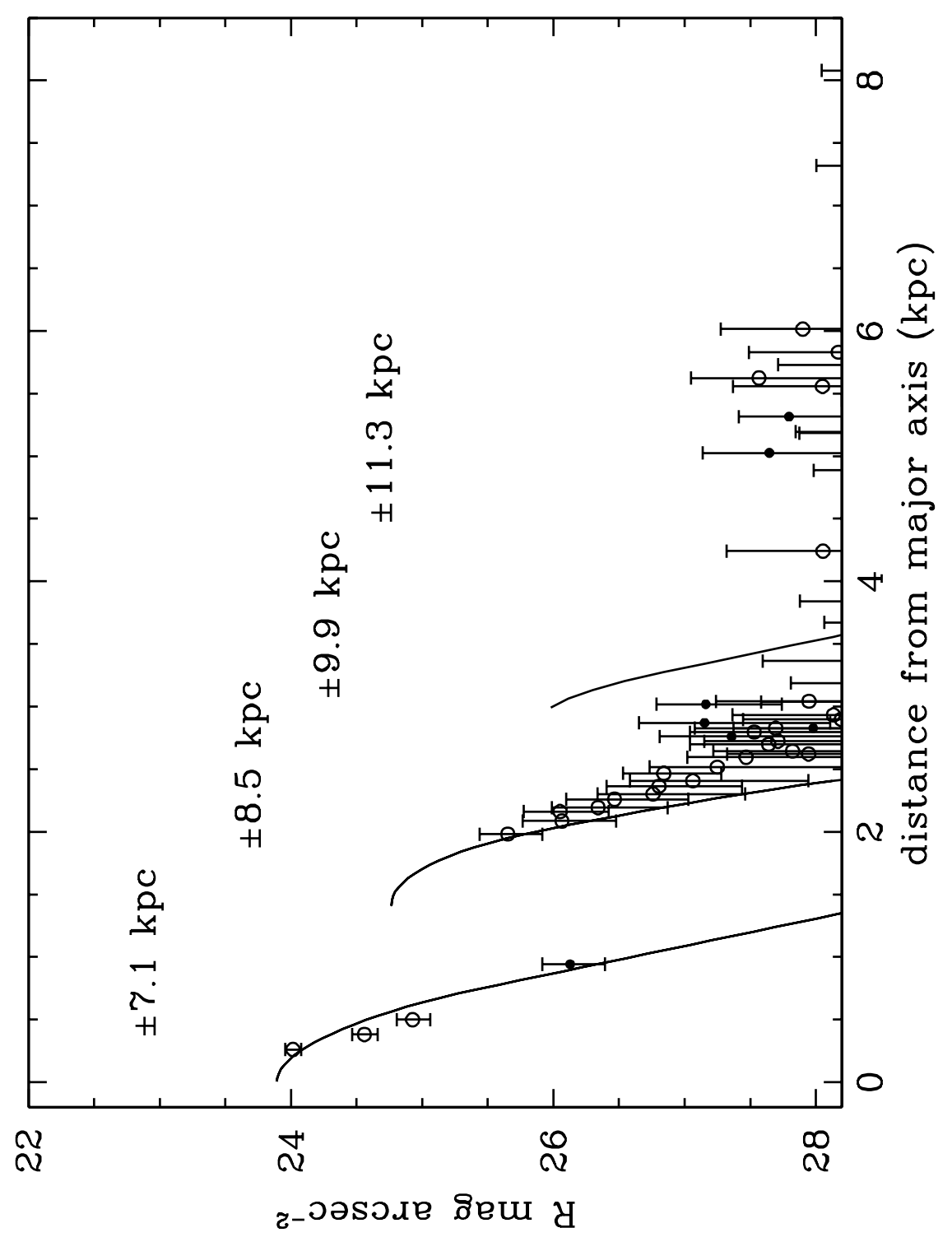

